\title{
EOSINOPHILIC FASCIITIS: CASE REPORT
}

Lizeth Chaparro Del Portillo' ${ }^{1, \star}$, Júlia Yoneshigue Laranja de Oliveira ${ }^{1}$, Hany Kelly Araujo Cruz¹, Bruno Bordalo Corrêa ${ }^{1}$, Luiza Catharina Brusasco Grandini ${ }^{1}$, Mario Zazur Perez ${ }^{1}$, Marcella Azevedo Borges Andrade ${ }^{1}$, Victor Berçot ${ }^{1}$, Nathalie Henriques Silva Canedo ${ }^{1}$, Catalina Collazos Castro ${ }^{1}$, Blanca Elena Rios Gomes Bica ${ }^{1}$

1.Hospital Universitário Clementino Fraga Filho, Rio de Janeiro (RJ), Brazil.

*Corresponding author: lizethchaparrodelportillo@hotmail.com

\section{BACKGROUND}

Eosinophilic fasciitis (EF) is a rare connective tissue disease, also known as Schulman disease, characterized by symmetrical and painful edema with progressive hardening and thickening of the skin, muscle fascia and subcutaneous cell tissue associated with inflammatory infiltrate, mainly lymphocytes and eosinophils. Groove sign and orange skin (peau d'orange) are characteristic cutaneous findings, related to this disease. In most cases, it is preceded by intense physical exercise or trauma. There is an association with peripheral eosinophilia, although it is not mandatory for the diagnosis of EF. Polyclonal hypergammaglobulinemia and high erythrocyte sedimentation rate are commonly present. Skin biopsy confirms the diagnosis; it must include deep tissues and muscle. High-dose glucocorticoids are the main treatment and can be associated with an immunosuppressive, as a sparing agent. The clinical case presented consists in EF with clinical picture, characteristic laboratory findings and biopsy confirmation.

\section{CASE REPORT}

A 52-year-old male noticed paresthesias in the left upper limb, mild edema, limited pinch movement and wrist arthralgia nine months previously to the diagnoses. He reported similar onset of contralateral limb and hardening of the skin, on the following months. Systemic symptoms, such as Raynaud's phenomenon, sclerodactyly, "pitting scars" or pneumopathy, were absent. No trauma nor exacerbated physical exertion have happened. On physical examination, bilateral skin thickening on the forearm was observed, forming the groove-shaped venous pathways when elevating the limbs (groove sign, Fig. 1). Hands and fingers were unaffected. The patient had the following complementary tests: eosinophils $1070 / \mathrm{mm} 3$ in peripheral blood; ESR: $87 \mathrm{~mm} / \mathrm{h}$, polyclonal hypergammaglobulinaemia and biopsy of deep skin and muscle showing perimysium inflammatory process with eosinophilic participation compatible with EF (Fig. 2). Prednisone $1 \mathrm{mg} / \mathrm{kg} /$ day treatment was instituted with favorable clinical response and rapid decline in eosinophilia.

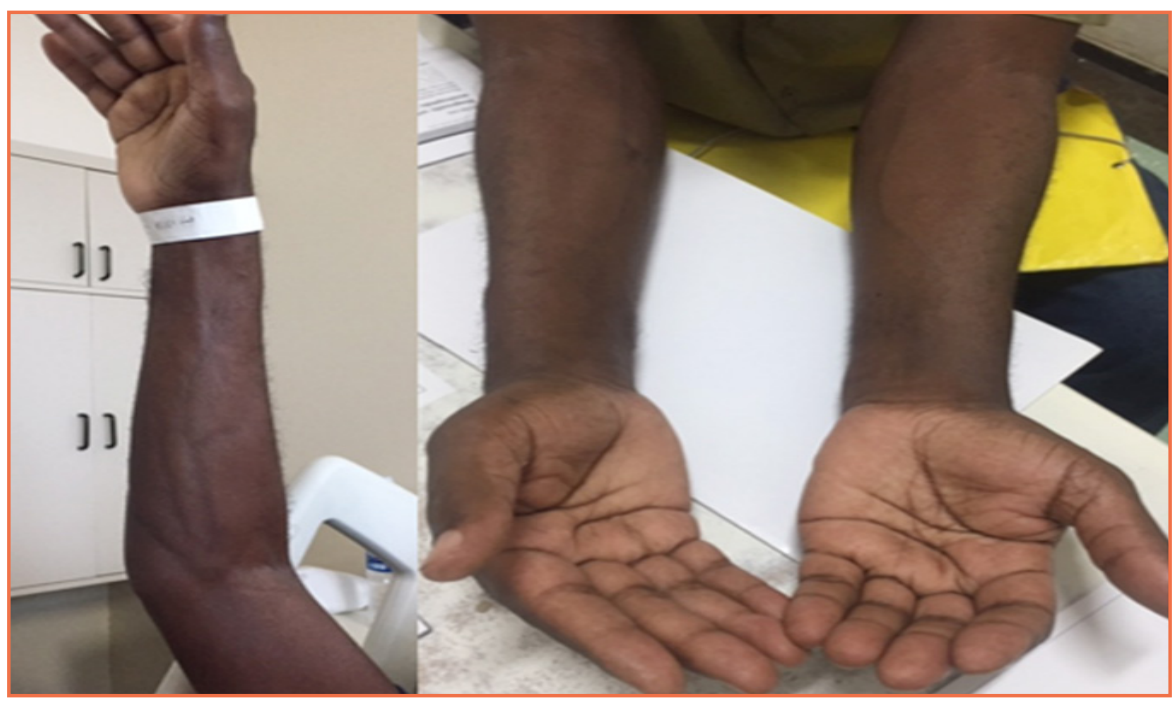

Figure 1. Groove sign. 

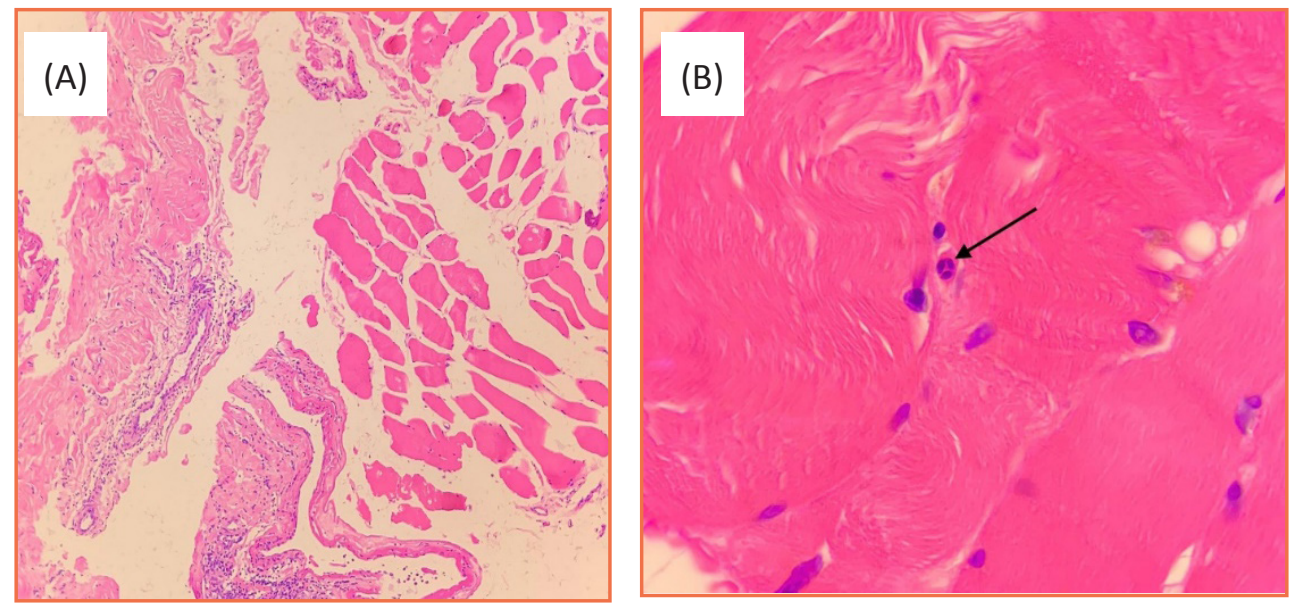

Figure 2. (A) Muscle fibers exhibit a predominantly perimisial inflammatory process with eventual participation of eosinophils (B), compatible with eosinophilic fasciitis.

\section{CONCLUSION}

Eosinophilic fasciitis is a rare connective tissue autoimmune fibrous disease of unknown etiology that affects men and women equally between third and sixth decades. Diagnosis is made by clinical and anatomopathological association. In many cases, diagnosis is delayed by ignorance of the disease, which leads to unnecessary invasive exams and inadequate treatments. Despite there is no consensual therapeutic strategy, oral corticosteroids remain the main treatment. The report above is essential to disseminate information regarding this rare clinical condition. 